\title{
Light's interaction with pigments in chloroplasts: The murburn perspective, with special relevance to carotenoids
}

\author{
Kelath Murali Manoj*, Afsal Manekkathodi \\ *Corresponding author, Satyamjayatu: The Science \& Ethics Foundation, \\ Kulappully, Shoranur-2 (PO), Palakkad District, Kerala State, India-679122. \\ Email: satyamjayatu@yahoo.com
}

\begin{abstract}
The prevailing understanding on the light reaction of oxygenic photosynthesis (photolytic photophosphorylation \& NADPH synthesis) considers the vast majority of the diverse pigments, chlorophyll binding proteins (CBPs) and light harvesting complexes (LHCs) as photon-energy relaying facets; only the chlorophyll $a$ couplets at the reaction center of the two photosystems I \& II are deemed to serve as photo-excitable electron emitters. Highlighting the historical perspectives involved, we present reasons why this conventional perception is unmet by theoretical foundations, unsupported by molecular awareness on the various pigments and unverified by physiological data available on chloroplasts. Further, we propose a simple diffusible reactive oxygen species (DROS)-based mechanism for correlating the functions of various light harvesting LHCs and CBPs with the reaction centers of Photosystems I \& II. The projected hypothesis may also provide a new perspective to photoreception research.
\end{abstract}

KEWORDS: Light reaction of photosynthesis; photolysis; photophosphorylation; chlorophyll; chloroplast; oxygenic photosynthesis; photosystem; light harvesting complex; antenna complex; murburn concept; photoreception 


\section{Historical progressions and current status}

Exploring the mechanism of photolysis and photophosphorylation is a tantalizing and enigmatic research field. The Editorial of the leading journal Science (Volume 340, Issue 6139, 21 June 2013, page 1373), while introducing two manuscripts within the particular issue, opines in the contents section- "It is unclear how energy absorbed by pigments in antenna proteins is transferred to the central site of chemical catalysis during photosynthesis." In a comprehensive review, Caffarri et al. (2014) say- "Although many aspects of the photosynthetic process are nowadays substantially elucidated, several details, specific regulations, and even structural details about photosynthesis in plants are still little known." In the abstract of a key structure elucidation paper, Wei et al. (2016) write- "The pathways along which excitation energy is transferred between them (reaction center, RC and light harvesting complexes, LHC), and their assembly mechanisms remain to be deciphered......" These statements strongly consolidate our assertion that there has been a dearth for a simple quantitative mechanistic rationale for the interaction of light with chloroplast pigments. Two anecdotes from the past are cited to show the historical relevance of our assertion.

1. Otto Warburg and Robert Emerson, sparred on some fundamental mechanistic aspects of the light reaction of photosynthesis. Warburg was a renowned biochemist, who won the Nobel prize and was nominated for same for a record 47 times. In the pertinent field, he is known for the Warburg effect, which concerns the lowered photosynthetic yield at high oxygen concentrations. Emerson (Warburg's doctoral student) had commissioned some original/pioneering quantitative studies in the field when he found that 2500 chlorophyll molecules form a photosynthetic unit. (Restated- maximum efficiency of oxygen formation resulted when one of $\sim 2500$ chlorophyll molecules absorbed a quantum of light.) He also championed the Emerson effect (Emerson et al., 1957), which proposed that two distinct photosystems existed in chloroplasts and these two distinct systems function in a synchronized physiological pathway for the acyclic photoylsisphotophosphorylation (Pl-Pp or photophos, in short). The bone of contention did not end with Emerson's untimely death in 1959; and Warburg sustained it until his own demise in 1970. The

discordance between the distinguished Warburg-Emerson teacher-disciple duo was on a very 
fundamental quantitative issue. While Warburg believed that 4 photons were enough to run the photolytic 4-electron acyclic scheme of oxygen evolution, Emerson believed that 8 photons were required for the same. Emerson's 8-photon perspective prevails today through the "linear electron transport of the Z-scheme".

2. The mechanism of "energy transfer" from the supposed LHCs and multitude of pigments scattered within the thylakoid membrane to the reaction center deep-seated within the photosystems has been a center of intense theoretical and experimental investigations. A pigment molecule within the photosystem or chlorophyll binding protein (CBP) or LHC could absorb a photon (corresponding to a quantum of energy) and the energy from this photon could be theoretically relayed to the reaction center (RC) Chl $a$ via two fundamental modalities-

(i) A radiative energy transfer by the emission of a photon of higher wavelength (e.g. by the phenomenon of fluorescence), which another chromophore could absorb and utilize.

(ii) A non-radiative and sort of conductive/convective energy transfer (a kind of dipoledipole quantized migration) of an "exciton" (one modality of which is the wellestablished Forster transfer).

The current mechanistic perspectives vouch that option (i) is rare but option (ii) is preponderant. However, the pioneer James Franck (of Franck-Condon principle fame) had argued in late 1930s that exciton transfers would not be possible within plant photosynthetic membrane systems (Franck \& Teller, 1938), owing to limitations posed by a non-organized lattice and slow kinetics. This was seconded by Albert Szent-Gyorgyi (Nobel laureate) even in the early 1960s (Avery et al., 1961). However, within a few years of publishing the paper above with Szent-Gyorgyi, Zoltan Bay made a volte face, and supported Robert Pearlstein, who advocated that exciton transfer was theoretically "viable" for photosynthetic energy relay (Bay \& Pearlstein, 1963). Their assumption of chlorophyll molecules being arranged in 3D lattices (rather than the 2D model used by Franck and Szent-Gyorgyi) was the game changer. In turn, this was apparently aided by the discovery of packing chlorophylls into 'stacks of thylakoid' within grana, which was supposed to enable this 3D navigation for excitons, and thus afford better rates. Their idea was subsequently supported by the experimental observation of exciton spectral lines at low temperatures with bacteriochlorophylls (Philipson \& Sauer, 1972). Thereafter, the consensus was that a series of exciton transfers or migrations (functionally equivalent to the transfer of energy 
quanta) are believed to occur before an actual electron transfer step takes place at the photosystems' reaction center. Currently, experts in the field indulge at least two distinct modalities of energy transfers (Panitchayangkoon et al., 2011) within the context above-

(a) Excitonic hopping [classical resonance energy transfer; wherein energy is transferred from one electronic state of a chromophore to the next. Herein, the "hops" indicate a rather particle type attribute that the energy has to assume to travel from its site of absorption along a random or predetermined route, to the destination of Chla-RC. It amounts to the transport of an electrically neutral "electron + electron hole".] AND

(b) Wave-like propagation [coherence transfer; wherein a quantum mechanical phase relationship exists/persists between the participants. Rather than behaving like a particle that travels in a given route, the energy packet absorbed behaves like "a quantummechanic wave-spread", thereby sampling all possible routes simultaneously or instantaneously.]

While some researchers of recent times believe that quantum coherence is the explanation for select experimental spectral observations, others have interpreted the observations with different theoretical basis or deemed them insignificant or irrelevant within the context (Engel et al., 2007; Nalbach et al., 2011; Tiwari et al., 2013; Ball, 2013; Halpin et al., 2014; Romero et al., 2014; Maiuri et al., 2018; Cao et al., 2020). It is evident that there is little consensus on the 'energy transfer' mechanism among the most erudite of the researchers in this field.

We believe that much of the confusions in the field resulted due to the belief that catalysis was restricted to enzymes' active sites. Further, majority of the relevant protein structures were not solved in the 1970s, by which time the mechanistic perceptions were 'frozen'. Furthermore, the proteins within the chloroplasts were seen as components of electron transport chains and chemiosmotic proton pumps; harnessing 'proton motive force' for ATP synthesis (Govindjee, 1975). The scenario is unchanged till date, as can be seen from the popular biochemistry textbooks (Lehninger et al., 2004; Berg et al., 2007; Voet \& Voet, 2011). Such views among the researchers denigrated diffusible reactive (oxygen) species or $\mathrm{DR}(\mathrm{O}) \mathrm{S}$ as wasteful toxic components and we have 'debunked' such ideas recently with the proposal of 'murburn concept' (Manoj et al., 2016a-c; Manoj, 2017; Manoj, 2018a; Jacob \& Manoj, 2019; Gideon et al., 2019; Manoj, 2020a). The new mechanism offers a radically new perspective for understanding the 
mechanistic redox chemistry at phospholipid interfaces in- (i) the endoplasmic reticulum or microsomal xenobiotic metabolism (mXM) within hepatocytes (Gideon et al., 2012; Parashar et al., 2014; Manoj et al., 2016b-c) and (ii) mitochondrial oxidative phosphorylations (mOxPhos) and thermogenesis (Manoj, 2017; Manoj, 2018a-b; Manoj et al., 2018; Manoj et al., 2019a-b; Manoj \& Bazhin, 2019; Manoj, 2020a-b; Manoj et al., 2020). We have also elucidated multiple structural and mechanistic similarities between the $\mathrm{mXM} / \mathrm{mOxPhos}$ systems and the chloroplast Pl-Pp machinery; and argued that fundamental metabolic format are similar in these systems (Manoj \& Bazhin, 2019; Gideon et al., 2020; Manoj, 2020c; Manoj et al., 2020a-e). Further, the structural details of chloroplasts and its various membrane-embedded components like photosystems (PS) and chlorophyll binding proteins (CBP)/light harvesting complexes (LHC) were revealed in greater details in recent times (Benson et al. 2015; Wei et al. 2016; Young et al. 2016; Mazor et al. 2017). Therefore, a revisit of the structure-function correlation and mechanism of membrane-embedded photolytic-phosphorylation (Pl-Pp) machinery of chloroplasts is mandated. With respect to these developments, we propose herein that the mechanistic insights we have ushered in redox biochemistry over the last decade could be useful in solving the underpinnings of light's interaction with chloroplastid pigments. Even in those systems, several spectroscopic evidences existed for some classical mechanistic outlooks, but we had argued that the in vivo/in situ conditions were quite distinct from those employed for spectroscopy and projected more facile ways for the physiological conditions. In this paper, we

first critique some standing perspectives and then present the basic framework of our ideas on the chloroplastid physiology of Pl-Pp.

\section{Critique on the energetics of the light reaction}

It is a daunting experimental task to accurately determine the functional efficiency of the coupling of Pl-Pp and Calvin-Benson cycle. This scenario exists because-

(1) It would be practically difficult to account for the actual number of photons of a given wavelength imparted to the leaf/chloroplast from any source of light and the number of quanta absorbed thereafter (within a given amount of time, in a given experimental setup). That is- we know that bright sunlight provides a source of radiation at $\sim 10^{21}$ photons $\mathrm{m}^{-2} \mathrm{~s}^{-1}$. But exactly how 
many of these photons are absorbed, and how do these absorptions vary with respect to the leaf thickness and position of the cell, etc. are issues that are very difficult to tabulate accurately.

(2) It would be practically difficult to trace the NADPH or ATP (and DROS) molecules formed in vivo or in situ because these products could be dynamically used up. Further, these products' formation route could be switched via cyclic, pseudocyclic and acyclic modalities. Therefore, the actual energy given into the system and the total product output formed by the system cannot be accurately computed experimentally. As a result, any treatments/arguments of practical values of efficiency shall not be discussed herein. (Also, fluorescence and luminescence based data/inferences are not dealt here because such experiments would be very "sensitive" to the DROS dynamics and therefore, subject to high levels of uncertainty.)

Across the visible spectrum, low absorption directly correlates to reduced photosynthetic rates. Approaching $700 \mathrm{~nm}$ (and beyond the same, wherein the chloroplasts show very little absorption), both the rate and efficiency drop to practically a zero value. The green light range (495-575 nm) gives low absorption and photosynthetic rates, concomitant with a relatively lesser quantum yield (Hogewoning et al., 2012). It is interesting to note that quantum yield retains a > $75 \%$ of maximal value across the visual spectrum, falling sharply at the red light. It has been estimated that most natural photosynthetic systems work holistically (conversion to total plant biomass with respect the total exposure to incident sunlight energy) at anywhere between 0.1 to $2 \%$ efficiency. If we were to discount for various types of losses at the input stage, some efficient plants (taken in optimal experimental setups) were found to give up to 6-8\% efficiency for the overall photosynthetic process/conversion.

Beginning with a set of traditional assumptions, a theoretical maximal value can be set for Pl-Pp. A mole of photons in the visible range (where plant chloroplasts are known to work!) can provide 171 to $299 \mathrm{~kJ}$, corresponding to the wavelengths of 700 to $400 \mathrm{~nm}$. The energy term for synthesizing a mole each of ATP and NADPH is $35 \mathrm{~kJ}$ and $220 \mathrm{~kJ}$ respectively. We know that a single Calvin-Benson cycle scheme requires $3 \mathrm{ATP}$ and $2 \mathrm{NADPH}$ (for the fixation of one molecule of $\mathrm{CO}_{2}$ ). As per the current beliefs, this ratio (3 ATP : $2 \mathrm{NADPH}$ ) happens to be the approximate estimation of products formed by the input of 4 electrons (from two different water molecules) into the double $\mathrm{Z}$ scheme (inclusive of Photosystems I and II), going through the 
acyclic modality. Now, let us suppose that PS I $(700 \mathrm{~nm})$ and PS II $(680 \mathrm{~nm})$ are perfectly synchronized and Einstein's photoelectronic laws apply. Then, 4 photons each of $440871 \mathrm{GHz}$ (4 x $176 \mathrm{~kJ} / \mathrm{mol}=704 \mathrm{~kJ} / \mathrm{mol})$ and $428274 \mathrm{GHz}(4 \times 171 \mathrm{~kJ} / \mathrm{mol}=684 \mathrm{~kJ} / \mathrm{mol})$ would be (minimally!) required to power the two photosystems for a 4-electron reaction. That would mean an energy input of $704 \mathrm{~kJ} / \mathrm{mol}$ at PS I and $684 \mathrm{~kJ} / \mathrm{mol}$ at PS II, equaling $\sim 1388 \mathrm{~kJ} / \mathrm{mol}$. The energy required to make the five "energy-rich" molecules is $\sim 545 \mathrm{~kJ} / \mathrm{mol}$ (ATP $=3 \times 35 \mathrm{~kJ} / \mathrm{mol}$ $=105 \mathrm{~kJ} / \mathrm{mol}$ and $\mathrm{NADPH}=2 \times 220 \mathrm{~kJ} / \mathrm{mol}=440 \mathrm{~kJ} / \mathrm{mol}$; and they add up to give $545 \mathrm{~kJ} / \mathrm{mol}$ ). That gives a maximal theoretical efficiency of $39 \%$ ( $=545 / 1388)$ for the overall Pl-Pp process. This is comparable with the experimentally determined maximal yield of 35-40\% that the most efficient/optimized man-made modern photovoltaic systems afford. In terms of the full photosynthetic cycle (overall coupling of Pl-Pp with Calvin-Benson cycle), since 48 photons are taken (at an average of $173.5 \mathrm{~kJ} / \mathrm{mol}$ ) for the formation of glucose, the efficiency runs down to $\sim 34 \%$. Therefore, if we assume that 8 photons going through a single $\mathrm{Z}$ scheme turnover gives 2 NADPH and 3 ATP molecules, the primary loss of energy occurs in the Pl-Pp system. The simple calculations presented herein counter commonly advocated presumptions that the light reaction of photosynthesis is a highly efficient process!

The sunlight-aided photosynthetic reaction occurring in chloroplasts can be minimally represented by: $\mathrm{CO}_{2}+\mathrm{H}_{2} \mathrm{O} \rightarrow \mathrm{CH}_{2} \mathrm{O}+\mathrm{O}_{2}$. The energy requirement for this reaction is $\sim 500$ $\mathrm{kJ} / \mathrm{mole}$. Depending on the wavelength of light within the visible spectrum $(300-170 \mathrm{~kJ} / \mathrm{mol}$ for the low and high wavelength ranges), this translates to a conservative requirement of 2-3 powering photons. Though the minimalistic equation above shows the formation of one molecule of oxygen from the reaction of a molecule each of $\mathrm{CO}_{2}$ and $\mathrm{H}_{2} \mathrm{O}$, Warburg knew that- (i) oxygen emitted in photosynthesis came from water, and (ii) Einstein's laws equated a photon to the emission of electron. Therefore, he perhaps stuck to the theoretical premise of a four electronic process considering the equation: $2 \mathrm{H}_{2} \mathrm{O} \rightarrow 4 \mathrm{H}^{+}+4 \mathrm{e}^{-}+\mathrm{O}_{2}$. However, Emerson's experimental orientations allowed him to make more realistic estimations. We know that breaking of the $\mathrm{O}-\mathrm{H}$ bond would need $\sim 464 \mathrm{~kJ} / \mathrm{mol}$, which is directly possible only by a photon of $\sim 257 \mathrm{~nm}$ (UV range) and we also know that 'water-breaking' happens even at visible wavelength spectrum. Therefore, it can be inferred that the "breaking of $\mathrm{O}-\mathrm{H}$ bonds in water" is an outcome of several 
indirect photochemical events within the chloroplasts. As per the current awareness of Z-scheme ETC and CRAS proposal, the equations for Pl-Pp are:

$2 \mathrm{H}_{2} \mathrm{O}+2 \mathrm{NADP}^{+} \rightarrow \mathrm{O}_{2}+2 \mathrm{NADPH}+2 \mathrm{H}^{+}\left(\mathrm{ZS}-\mathrm{ETC}: \Delta_{\mathrm{r}} G_{\mathrm{aq}}^{\mathrm{o}}=1355 \mathrm{~kJ} / \mathrm{mol}\right)$

$3 \mathrm{ADPOH}+3 \mathrm{POH} \rightarrow 3 \mathrm{ADPOP}+3 \mathrm{H}_{2} \mathrm{O}\left(\mathrm{CRAS}: \Delta_{\mathrm{r}} G_{\mathrm{aq}}^{\mathrm{o}}=109 \mathrm{~kJ} / \mathrm{mol}\right)$

However, in the classical scheme, four photons each of $680 \mathrm{~nm}(176 \mathrm{~kJ} / \mathrm{mol})$ and $700 \mathrm{~nm}(171$ $\mathrm{kJ} / \mathrm{mol}$ ) musters only $\sim 1390 \mathrm{~kJ} / \mathrm{mol}$, which is significantly short of the required energetic term of $1464 \mathrm{~kJ} / \mathrm{mol}$ needed for the overall uphill reaction (as shown above). This is when the energy required for trans-membrane proton pumping is unaccounted in the calculation and if that is also considered, it would only make the conventional explanation even more unviable. Therefore, both the proponents of Z-scheme and Warburg were misplaced in accounting the light reaction energetics. Other critical aspects of energetics are discussed elsewhere (Manoj \& Bazhin, 2019; Manoj et al., 2020b,c,e).

The electron transfer process from the reaction center chlorophyll to say, an agent like quinone, is fraught with significant loss. This is essential so that the process becomes significantly downhill. If the $\mathrm{Chl}^{*} \rightarrow \mathrm{Chl}^{+\cdot}$ half reaction has a mid-point potential of $\sim-1000 \mathrm{mV}$ and the twoelectron quinone redox couple has a mid-point potential of $\sim-45 \mathrm{mV}$. Using the equation $\Delta G \boldsymbol{G}^{\circ}=$ $\boldsymbol{- n F \Delta E}$ ', the free energy change of electron transfer between the two couples gives a "nontrappable" value of $-180 \mathrm{~kJ} / \mathrm{mol}$ (practically, the whole quantum absorbed from light!). Then, it is difficult to explain how such an ETC can work at a much greater efficiency, if the overall theoretical efficiency is supposed to be at 39\%! Clearly, the theoretical benchmark and the conceived practical calculations (based on an assumed mechanistic pathway) do not match.

\section{Critical comments on 'excitons' and 'coherence' in chloroplast physiology}

Englemann had originally shown with an elegant experiment that the wavelengths of light most efficient for Pl-Pp falls at the two ends of the visible spectrum- "violet" and "red" regions. The action/absorption spectrum shows little correlation with the relatively consistent quantum yield (photosynthesis efficiency) across the extended region of 400-700 nm (Figure 1). This is observed when the chloroplasts have a preponderence of chlorophyll pigments that 
absorb/function around the red region. The prevailing "energy" relay systems cannot explain the overall energy capture or harnessing phenomenon, if the electron emission only occurs at PS I and PS II reaction centers, with the unique pigment of Chla (which absorb "functionally" only at 680-700 nm). The current schemes cannot reason why: (i) Chlorophyll $a$ (which has very sharp absorption bands and which are the ultimate receiver of energy quanta at the photosystem complexes that carry out charge separation) should be in excess of Chlorophyll $b$ (with wider absorption range and which are the purported primary collectors of energy quanta) and (ii) PS II should be more than PS I when Z-scheme dictates a 1:1 connectivity as per the visual depiction of electron connectivity. Therefore, it remains a conundrum as to why should there be so many pigments presented in diverse orientations. The random arrangements of LHCs and CBP leave little scope for any deterministic mechanism's operation, and the working scheme must to stochastic/statistical.

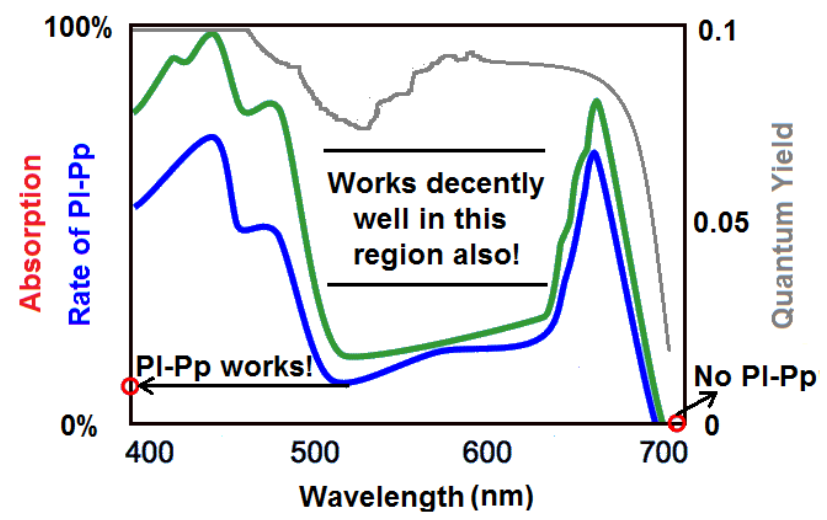

Figure 1: A schematic representation of the absorption and photosynthetic efficiency across the visual spectrum of light. (Unabashedly adapted from Ross Koning's website, http://plantphys.info/plant_physiology/light.shtml) The left Y-axis is drawn on an arbitrary but relative scale. Experimentally, the minimal/optimal value of $\sim 10$ photons (average of 8 to 12) is needed per oxygen molecule evolution, which is represented as the maximal value on the right $Y$ axis. Chla absorbs light at $430 \mathrm{~nm}$ and $662 \mathrm{~nm}$ whereas Chlb absorbs light at $453 \mathrm{~nm}$ and 642 $n m$. These abundant pigments absorb in the blue and red wavelength ranges, and therefore, the leaves appear green. Carotenoids absorb between $460 \mathrm{~nm}$ and $550 \mathrm{~nm}$ and appear orange-red.

It must be considered that all known matter $\leftrightarrow$ matter and radiation $\leftrightarrow$ matter interactions and transfer processes incur loss of energy. That is, if any radiation interacts with matter, and if it alters the state of the matter in any way, it is accompanied with a change in energy term of both, radiation and matter. Further, the efficiency of energy transfers between any two molecules would be critically dependent on distances and orientations. The proposal that a loss-less transfer 
of energy quantum between homo/hetero species, within a non-crystalline, heterogeneous, multiphasic environment of biomolecular systems "randomly embedded" in lipids and water appears to be a very fantastic/deterministic proposal indeed. For achieving electron transfers by plastoquinone and plastocyanin, the system is deemed highly fluidic (enabling high motility of electron relay agents), but to account for the exciton transfers, the very same system is deemed to be in solid state. Such assumptions are mutually incompatible.

Exciton interactions are appreciable and measurable only for short distances of $<20 \AA$ for molecules like chlorophyll ( $\sim 5$ times porphyrin's thickness and less than twice the diameter, depending on the ambiance). In contrast, Forster resonance interaction may be observed $<100 \AA$. But then, Forster energy transmission is not efficient. Therefore, the purported scheme of energy transfer, whether via exciton/quantum coherence, from LHC II to LHC I / CBP (chlorophyll binding protein) to reaction center Chl $a$ (some researchers project the following scheme: from $\mathrm{Chl} b \rightarrow$ Lutein $\rightarrow$ Zeaxanthin $\rightarrow \beta$-Carotene $\rightarrow$ Lycopene $\rightarrow$ Chl $a$ RC, of P680 or P700), does not explain the action spectrum versus absorption spectrum juxtaposition, that too, at a fantastic $>90 \%$ efficiency, in a timescale of $10^{-10} \mathrm{~s}$. Across the bacterial and plant systems, the number of elements in a photosynthetic unit (the number of pigments like chlorophyll involved in the absorption of definite light quanta, leading to the evolution of a single oxygen molecule) does not conform and are found to vary from a few tens to a few thousands (50-5000; Emerson found the average number to be $\sim 2500$ ). This clearly shows that the underlying operable mechanism is statistical/stochastic. The light-induced electron transfer is supposed to occur only at the reaction centers of PS I and PS II. The current Z-scheme of " 8 photon - 2 water molecules - 1 molecule of oxygen (4 protons)" translates to an approximate value of $\sim 2400$ chlorophylls per oxygen molecule evolved, $\sim 600$ molecules of chlorophylls per electron transferred and $\sim 300$ chlorophyll molecules per photon absorbed. Why such superfluous numbers of pigments and more importantly, why are they present rather randomly across the thylakoid membranes? The plant LHC and CBP hardly have any repetitive or ordered structure like that of the bacterial pigment system arrays. Given these perspectives, it is really unforeseeable how such systems could trap and relay photon energy without loss. 
Thylakoid membranes are amorphous, heterogeneous and anisotropic semi-fluidic structures surrounded by aqueous pools. The theory of exciton migration was originally developed for relatively crystalline/homogeneous/isotropic molecular assemblies, although in recent times, contrary evidence has been reported (Messina et al., 2010). The evidence for heterogenous energy transfer arises from the ascribing of Chla fluorescence bands to absorption in wavelengths where Chla has little absorption (whereas other pigments absorb strongly). It is difficult to envisage homo and hetero energy transfers between various pigments at high efficiencies (with purported values even reaching $100 \%$ ). When the energy transfer efficiency from carotenoids to Chla is $10-50 \%$, one cannot explain the action spectrum of photosynthesis. When both the lifetime of excited pigments and fluorescence timeframes fall under the range of nanoseconds, the natural fluorescence would effectively compete with exciton transfer mechanism. Using the observation that a polarized excitation light resulted in depolarized fluorescence as an evidence for homogeneous energy transfer could be erroneous, if DROS were involved as a transient agent within the reaction system. There exists little evidence to support the migration or transfer of electron-hole in the photosynthetic units. There is no evidence to support the notion that chlorophyll is present in the membranes under a crystalline environment. Neither the absorption nor fluorescence is strongly polarized, indicating that the pigments are not ordered in any simple periodic lattices (p.s. some bacterial systems may be exceptions to the above statement). While the fluorescence yield is high for chlorophyll in solution, it gives almost an order lesser yield in situ. For a molecule like chlorophyll, exciton interactions are appreciable and measurable only for distances less than $2 \mathrm{~nm}$ (a few folds of its linear dimensions). These arguments suggest that diverse chlorophyll molecules are subject to different microenvironments. In such scenarios, it is inappropriate to extrapolate the results found with ordered pigments' photosynthetic structures found in cyanobacteria to that of the random array in plants (Engel at al., 2007). In this context, one must reflect the opinion of Duan et al. (2017), "The conditions under which matter displays quantum features and biological functionality are contrarious." Therefore, we should look for simpler models to explain photosynthesis, and it would be premature to suppose that plants are capable of quantum computing. Therefore, to reason for an apparently high efficiency for the relay of photons (and their energy) absorbed, we counter the earlier proposals that plant cells employ quantum computations (Engel et al., 2007; Biello, 2007). 
Forster energy transfer occurs between molecules 2 to $10 \mathrm{~nm}$ apart and for most systems with good orientations, the Forster distance (the spatial separation between the donor and acceptor which yields $50 \%$ energy loss in transmission) is $\sim 5 \mathrm{~nm}$. The multi-protein photosystems' core (not inclusive of the LHCs) measures about $10 \mathrm{~nm}$ or so, and they are separated from each other (homo or hetero distances) by about the same or double that distance or even more. The LHCs dimensions are similar to the photosystem core dimensions but they may be more closely packed with respect to themselves and to the photosystems. The distance between any two photoreceptors (say chlorophyll molecules) is about 6-7 nm, in the least. These findings/arguments lead to a forthright inference- energy quanta transmission in such systems cannot be efficient! Also, Anderson localization would not permit diffusion of waves in a disordered medium like chloroplasts. Therefore, we find resonance with the theoretical cum experimental work from Duan et al (2016) has an explicit title- "Nature does not rely on longlived electronic quantum coherence for photosynthetic energy transfer." We believe that the mechanism of photon processing and 'energy relay' remains muddled because we may be looking at the picture with a wrong perspective.

\section{Re-interpretation of bacterial rhodopsin sponsored trapping of light energy}

Herein, we deal with bacterial rhodopsin, which is taken as a model for light-driven proton pumps (a simpler model of the same types as the chloroplast ETC). Rhodopsin is a rather simple protein (a monomer) with seven trans-membrane helices. The photosensitive pigment or redox cofactor in rhodopsin is a simple molecule, retinal. The current mechanism for retinal-based photo-induced processes involve cis-trans isomerizations (and associated conformational change in the protein), leading to specific amino acid residues' protonations and deprotonations. These processes are supposed to lead to a proton pumping effect. Several research groups (working on different rhodopsins in different time periods) have found that at physiologically relevant regimes (at slightly above neutral $\mathrm{pH}$, with moderate ionic strength), the $\mathrm{pK}_{\mathrm{a}}$ of lysine conjugated retinal Schiff's base ranges from a value of 10 to 16, with most groups placing it to 13 (Druckmann et al., 1982; Sheves et al., 1986; Balashov et al., 1991; Steinberg et al., 1993; Liang et al. 1994; Govindjee et al. 1994; Rousso et al., 1995)! It is highly unlikely that this 
species would impart a proton to the side-chain of an aspartate residue (as the currently prevailing mechanistic interpretation solicits!) or any other species at physiological $\mathrm{pH}$ ranges. With the available understanding of chemico-physics, it is improbable that such a protein-based mechanism could serve as trans-membrane proton pumps.

Therefore, when seeing that (under sub-micron dimensional regimes)- (i) there are no source of free protons, (ii) there are little ways that a proton gradient can be efficiently tapped by a biological system, and (iii) the mOxPhos proton pump model is untenable- it would make a lot of chemical sense to seek an alternate explanation for the mechanism of rhodopsin functioning. It appears more likely that the covalent modification with lysine is enabled to tether the retinal into the protein within the trans-membrane region (to enable murcat process) and enhance the electron density even fruther by conjugating the sulphur lone pairs into the system.

Our alternative hypothesis in the photochemistry of carotenoids: Retinal is a pi-conjugated system, known well to get excited by light, and also known to generate reactive species by a diverse array of envisaged/probable mechanisms (Aboltin et al., 2013). Retinal can be excited by light to give its excited form. The essential process is represented by-

$\mathrm{R}+h v \rightarrow \mathrm{R}^{*}$

The excited or native species can lose/gain an electron and/or thus lead to several transiently stabilized species within the system.

$\mathrm{R}^{*} \rightarrow \mathrm{R}^{+}+\mathrm{e}^{-}$

$\mathrm{R}$ or $\mathrm{R}^{*}+\mathrm{e}^{-} \rightarrow \mathrm{R}^{*-}$

[The formation and dynamics of aquated electron in photochemical systems is well-known and its implication in photosynthesis was speculated even in the 1960s (Schindewolf, 1968). In oxygenated systems, aquated electron would react instantly to give superoxide $\left(\Delta \mathrm{G}^{\mathrm{o}},=-249.6\right.$ $\mathrm{kJ} / \mathrm{mol}$ (Manoj \& Bazhin, 2019), with a rate constant of $2 \times 10^{10} \mathrm{M}^{-1} \mathrm{~s}^{-1}$ (Takahashi et al., 1988).] Else, it can also crossover to triplet state and react with oxygen.

$\mathrm{R}^{*} \rightarrow{ }^{3} \mathrm{R}^{*}$

${ }^{3} \mathrm{R}^{*}+{ }^{3} \mathrm{O}_{2} \rightarrow \mathrm{R}+{ }^{1} \mathrm{O}_{2}$ 
[Long-standing researchers in the field would know that F. K. Fong had proposed a convincing theory for a similar process for chlorophylls, which is aided by the parallel positioning of the two RC chlorophylls within the photosystems (Fong, 1974).]

As evident, in such an oxygenic system, superoxide could be formed in a multitude of ways.

$\mathrm{O}_{2}+\mathrm{e}^{-} \rightarrow \mathrm{O}_{2}^{\cdot-}$

$\mathrm{R}^{*}+\mathrm{O}_{2} \rightarrow \mathrm{R}^{\cdot+}+\mathrm{O}_{2}^{\cdot-}$

$\mathrm{R}^{\cdot-}+\mathrm{O}_{2} \rightarrow \mathrm{R}+\mathrm{O}_{2}^{\cdot-}$

Thereafter, within a partially closed and heterogenous system (with specific transport/partioning principles), directional electron transfer could spontaneously set in place, yielding continuous source of superoxide species. In the presence of adequate protons (and favored by reductant), the dismutation of superoxide $\left(\mathrm{pK}_{\mathrm{a}}\right.$ is on the acidic scale) gives peroxide $\left(\mathrm{pK}_{\mathrm{a}}\right.$ is on the alkaline scale) and the murburn chemistry that results can result in small amounts of hydroxyl radicals. [We have also argued that electron abstraction from hydroxide ions and water molecule (only PS II) can source hydroxyl radicals, which occurs at the two photosystems. So, the LHC and other associated CBPs and randomly distributed pigments are a source of superoxide whereas the photosystems are a source of hydroxyl radical. Only the hydroxyl radical acts as an efficient switch to drive the photolysis forward.] These transitions and reactions lead to "breaking" of water and release of protons in the milieu. The site of proton release would depend on the orientation of the retinal moiety within the trans-membrane region of the rhodopsin molecule (and the accompanying relay networks of the amino acid residues that ensconse the retinal molecule) and the extent of ROS detection in the milieu would be dependent on the efficiency of the quenching or cycling systems around. This phenomenon could also be inadvertently or indirectly referred to as a "proton pump". These DROS based mechanisms also gain significance from an initial state considerations. The small organelles lack adequate amounts of protons for the excess of proteins to work! This aspect has been discussed in earlier works from our group (Manoj, 2017; Manoj, 2018). Therefore, the simple chemical logic of explaining rhodopsin's role in sponsoring ATPsynthase activity also accounts for Jagendorf's or Racker's experiments of the past (Jagendorf \& Uribe, 1966; Racker \& Stoeckenius, 1974) and those like Otrin et al's experiment (Otrin et al., 2017) in recent times. It is very evident that the common element in all these systems is DROS and therefore, the operational feasiblity of murburn concept is imminent. 
This inference implies that the LHCs and other pigments within the Pl-Pp system would have new functionalities that could aid light energy capture associated with electron transfer processes.

The updated information on structures of photosystems, CBPs, LHCs, supercomplexes and pigment distribution in chloroplasts (Croce and Amerongen, 2020) show that the multitudes of accessory pigments are juxtaposed to the reaction center (RC) chlorophyll $a$ doublet at various distances and in diverse configurations. The multitudes of pigments are usually present without any covalent tethering to the proteins. These findings signify an absence of strong evolutionary logic for deterministic electron tunneling routes within the protein or energy relay between them; also suggesting the relevance of a stochastic mechanism like that of murburn concept. The charge separation occurs at the RC in ps and the energy transfers/decays of excited states are in the time range of ps to ns. Then, one can pose three queries (besides several others!) that cannot be met by the classical scheme: (i) What serves as the source of electrons to PS I in such time frames, particularly in systems with knocked out plastocyanin (wherein a practically unaltered physiological outcome is observed)? (ii) As PS II are predominantly present in the deep-seated appressed grana whereas Cyt. $b_{6} f$ are located in peripheral thylakoids, how could the electron relay of reduced plastoquinone jump the stacks of membranes? (iii) How is the achievement of steady-state oxygen evolution in the Kok-Joliot cycle explained, wherein the oxygen evolution maximizes after four or five cycles of periodic exposure to light, when the spacing of excitation is at much higher timescales? These facts do call for the involvement of diffusible species as

electron relay agents within the system. Further, the clumping of CYPs in endoplasmic reticulum (Parashar and Manoj, 2020) and formation of supercomplex structures in mitochondria (Manoj et al., 2019b) were shown to be an evolutionary measure that aided DROS-dynamics and DROSderived outcomes. Similarly, in the chloroplast machinery, the proteins' structures also have been shown to aid DROS dynamics (Manoj et al., 2020).

\section{The murburn scheme of LHC/CBP and photosystems' interaction with light}

We accept that "non-route chlorophylls and carotenoids" scattered around the membrane and within the membrane-embedded proteins could relay energy by simple fluorescence or Forster 
transfer mechanisms. This mechanism would lead to some 'energy transfers' to RC. However, the major components of photo-active pigments work via the scheme depicted in Figure 2, which is an extended form of our earlier proposal (Manoj et al., 2020e). The murburn reactions of ATP synthesis (Manoj et al., 2019a,b; Manoj \& Bazhin, 2019) and reduction of $\mathrm{NADP}^{+}$(and its oxidation by photorespiration) occur simultaneously. LHCs and CBPs feed and drain from the oxygen-superoxide pool, around the thylakoids. (Only photolytic oxygenesis would show up as oxygen generated. The dynamic and catalytic utilization of oxygen via LHCs/CBPs would not show in the overall tally because oxygen would be regenerated upon its own reduction or oxidation.) Therefore, the murburn scheme explains the formation of oxygen, ATP and NADPH with simple chemical reactions alone, without invoking proton pumps and seeks only small contributions from fluorescence and exciton transfers (coherence phenomena can be safely deemed as inoperative). The two photosystems' synergy is better explained via the parallel system. Also, Cyt. $b_{6} f$ 's 'turbo role' to reset one-electron redox equivalents from the membrane phase can be visualized by the DROS machinery here. The interactive scheme of DROS effectively synthesizes ATP and utilization of NADPH can be explained by the photorespiration activity of Compound I like NADPH-dehydrogenase (NDH) and the recharging of electrons to oxygen. Essentially, the system works by utilizing oxygen (both existing and generated) as a catalyst. That is- it is a simple photoelectrical system wherein an $\mathrm{O}-\mathrm{H}$ bond is lysed in the uphill reaction (using light) to generate electrons and oxygen is voided through a series of steps (exhaust!) and the same O-H bond is made (in the downhill phase) to thermodynamically drive ATP synthesis, thermogenesis and organelle homeostasis (Manoj et al., 2019a,b; Manoj \& Bazhin, 2019). This regenerative and self-sustaining logic is one of the simplest ideas of the 'cell-machine', which generates a recycling chemical catalytic strategy to do 'work'. 


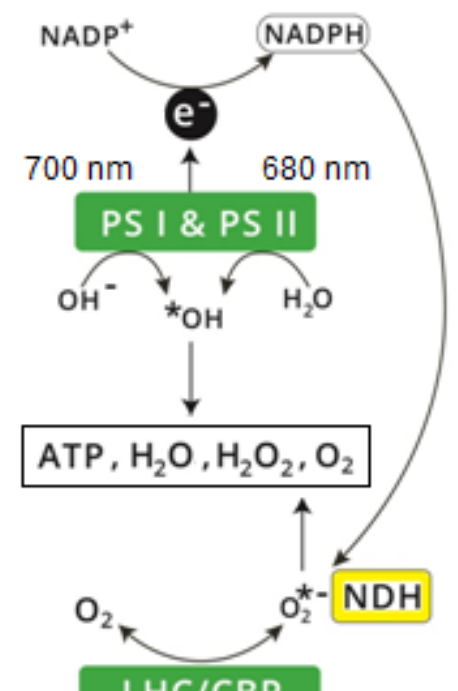

\section{LHC/CBP}

Figure 2: The basic murburn reaction scheme of oxygenic photolytic phosphorylations.

For details of the reactions, please refer our precepts papers for aerobic respiration (Manoj \& Bazhin, 2019) and oxygenic photosynthesis (Manoj et al., 2020e). Our proposal is supported by the fact that all components of the chloroplast machinery: Photosystems I \& II, Cyt. $b_{6} f$, plastocyanin, ferredoxin (Fd), Fd-NADP reductase, LHCs/CBPs are known to be involved in DROS production and dynamics (Khorobrykh et al., 2020; Foyer, 2018; Sarwar Jahan et al., 2016; Xu et al., 2012). Also, all classes of the pigments involved (chlorophylls, carotenoids, etc.) are known to be both photo- and redox- active, interacting with DROS (Konovalova et al., 1997; Han et al., 2012; Trchounian et al., 2016). The murburn proposal's coupled and minimalized mass-charge balanced equation (the practical equation would vary owing to the stochastic nature of the reaction outcome) for Pl-Pp involves the obligatory intermediacy and production of DROS:

$4 \mathrm{OH}^{-}+3 \mathrm{NADP}^{+}+\mathrm{ADPOH}+\mathrm{POH}+\mathrm{H}^{+} \rightarrow \mathrm{O}_{2}+3 \mathrm{NADPH}+\mathrm{ADPOP}+\mathrm{H}_{2} \mathrm{O}+\mathrm{H}_{2} \mathrm{O}_{2} ; h v>1847 \mathrm{~kJ} / \mathrm{mol}$

The proposal correctly approximates the bond energetics for four O-H bonds' $(4 \mathrm{x} 464=1856$ $\mathrm{kJ} / \mathrm{mol}$ ) breakage. (One molecule of NADPH can give several molecules of ATP, using the photo-respiration machinery like NDH, as shown in Figure 2.) Therefore, the murburn proposal appeals from several angles of reaction chemistry.

\section{Conclusions}


In the light of the new mechanistic explanations and structural awareness on plant chlorolast architecture and known structures and distribution of photosytems/LHCs/CBPs, it is opportune to revisit the mechanism of light's interaction with the photosynthetic machinery. The murburn proposal floated is a thermodynamically and kinetically viable hypothesis, well supported by architecture of chloroplasts, known nature of the redox active light sensitive pigments, nature and mobility of oxygen, observation of DROS in steady state, non-specificity of electron transfers, DROS-mediated ATP synthesis observed in chloroplasts, etc. Further experiments are needed to ratify the new proposal and chart out the details. We project that the murburn model could also form a basis for re-interpretation of the photoreceptive phenomena for the retinoid cycle of the opsin containing rod cells in retina. For, while the traditional explanation rests on the protein's conformation based on a cis-trans configuration change, the murburn perspective offers a direct electronic/electrical response that could be relayed to the optic nerve connectivity.

Declarations: The work was powered by Satyamjayatu: The Science \& Ethics Foundation. The authors have no conflict of interests to declare. We thank Vivian David Jacob for Figure 2.

\section{REFERENCES}

Aboltin PV, Shevchenko TF, Shumaev KB, Kalamkarov GR. Photoinduced production of reactive oxygen species by retinal derivatives and conjugates. Biofizika 2013; 58:246-251.

Avery J, Bay Z, Szent-Gyorgyi A. On the energy transfer in biological systems. Biochemistry 1961; 47:1742-1744.

Balashov SP, Govindjee R, Ebrey TG. Redshift of the purple membrane absorption band and the deprotonation of tyrosine residues at high $\mathrm{pH}$ : Origin of the parallel photocycles of transbacteriorhodopsin. Biophys J. 1991; 60:475-490.

Ball, P. Is photosynthesis quantum-ish? Physics World (IOP Publishing), April (2018).

Bay Z, Pearlstein RM. A theory of energy transfer in the photosynthetic unit. Proc Natl Acad Sci USA. 1963; 50:1071-1078.

Berg JM, Tymoczko JL, Stryer L. Biochemistry $6^{\text {th }}$ Edition. 2007, WH Freeman, New York.

Benson SL, Maheswaran P, Ware MA, et al An intact light harvesting complex I antenna system is required for complete state transitions in Arabidopsis. Nat Plants 2015; 1:15176.

Biello D. When it comes to photosynthesis, plants perform quantum computation. Scientific American, 2013; April 13.

Caffarri S, Tibiletti T, Jennings RC, Santabarbara S. A comparison between plant photosystem I and photosystem II architecture and functioning. Curr Protein Pept Sci. 2014;15:296-331.

Cao J, et al. Quantum biology revisited. Science Adv. 2020; 6:eaaz4888. 
Croce R, van Amerongen H. Light harvesting in oxygenic photosynthesis: Structural biology meets spectroscopy. Science 2020; 369(6506):eaay2058.

Druckmann S, Ottolenghi M, Pande A, Pande J, Callender RH. Acid-base equilibrium of the Schiff base in bacteriorhodopsin. Biochemistry 1982; 21:4953-4959.

Duan H-G, Prokhorenko VI, Cogdell RJ, et al. Nature does not rely on long-lived electronic quantum coherence for photosynthetic energy transfer. Proc Natl Acad Sci U S A. 2017; 114:8493-8498.

Emerson R, Chalmers RV, Cederstrand CN. Some factors influencing the long wave limit of photosynthesis. Proc Natl Acad Sci USA.1957; 43:133-143.

Engel GS, Calhoun TR, Read EL, et al. Evidence for wavelike energy transfer through quantum coherence in photosynthetic systems. Nature 2007; 446:782-786.

Fong FK. Energy upconversion theory of the primary photochemical reaction in plant photosynthesis. $J$ Theor Biol. 1974; 46:407-420.

Foyer GH. Reactive oxygen species, oxidative signaling and the regulation of photosynthesis. Env Exp Bot. 2018; 154:134-142.

Franck J, Teller E. Migration and photochemical action of excitation energy in crystals. J Chem Phys. 1938; 6:861-872.

Gideon DA, et al., What is the functional role of N-terminal transmembrane helices in the metabolism mediated by liver microsomal cytochrome P450 and its reductase?, Cell Biochem Biophys. 2012; 63:3545 .

Gideon DA, Jacob VD, Manoj KM. 2020: Murburn concept heralds a new era in cellular bioenergetics, Biomed Rev. 2019; 30:89-98.

Gideon DA, Nirusimhan V, Manoj KM. Are plastocyanin and ferredoxin specific electron carriers or generic redox capacitors? Classical and murburn perspectives on two photosynthetic proteins. J Biomol Str Dyn. 2020; doi:10.1080/07391102.2020.1835715

Govindjee R, Balashov S, Ebrey T, Oesterhelt D, Steinberg G, Sheves M. Lowering the intrinsic $\mathrm{pK}_{\mathrm{a}}$ of the chromophore's Schiff base can restore its light-induced deprotonation in the inactive Tyr-57--> Asn mutant of bacteriorhodopsin. J Biol Chem. 1994; 269:14353-14354.

Govindjee. Bioenergetics of photosynthesis. Academic Press, New York, 1975.

Halpin J, et al. Two-dimensional spectroscopy of a molecular dimer unveils the effect of vibronic coupling on exciton coherences. Nature Chem. 2014; 6:106-201.

Han R-M, Zhang JP, Skibsted LH. Reaction dynamics of flavonoids and carotenoids as antioxidants. Molecules 2012; 17:2140-2160.

Hogewoning, SW et al. Photosynthetic quantum yield dynamics: from photosystems to leaves. Plant Cell. 2012; 24:1921-1935.

Jacob VD, Manoj KM. Are adipocytes and ROS mistaken for villains, or are they protagonists in the drama of life? The murburn perspective. Adipobiology 2019; 10:7-16.

Jagendorf AT, Uribe E. ATP formation caused by acid-base transition of spinach chloroplasts. Proc Natl Acad Sci USA. 1966; 55:170-177.

Khorobrykh S, Havurinne V, Matilla H, Tyystjarvi E. Oxygen and ROS in photosynthesis. Plants 2020; 9:91.

Konovalova TA, Kispert LD, Konovalov VV. Photoinduced electron transfer between carotenoids and solvent molecules. J Phys Chem B 1997; 101:7858-7862. 
Lehninger AL, Nelson DL, Cox M. Principles of Biochemistry. 2004; Palgrave Macmillan Limited, New York.

Liang J, Steinberg G, Livnah N, Sheves M, Ebrey TG, Tsuda M. The $\mathrm{pK}_{\mathrm{a}}$ of the protonated Schiff bases of gecko cone and octopus visual pigments. Biophys J. 1994; 67:848-854.

Maiuri $\mathrm{M}$ et al. Coherent wavepackets in the FBO complex are robust to electronic-structure perturbations caused by mutagenesis. Nature Chem. 2018; 10:177-183.

Manoj KM, et al. Atypical profiles and modulations of heme-enzymes catalyzed outcomes by low amounts of diverse additives suggest diffusible radicals' obligatory involvement in such redox reactions. Biochimie 2016a; 125:91-111.

Manoj KM, et al. Electron transfer amongst flavo- and hemo-proteins: diffusible species effect the relay processes, not protein-protein binding. RSC Adv. 2016b; 6:24121-24129.

Manoj KM, Parashar A, Gade SK, Venkatachalam A. Functioning of microsomal cytochrome P450s: murburn concept explains the metabolism of xenobiotics in hepatocytes. Front Pharmacol. 2016c; 7:161.

Manoj KM. Debunking chemiosmosis and proposing murburn concept as the explanation for cellular respiration, Biomed Rev. 2017; 28:35-52.

Manoj KM. The ubiquitous biochemical logic of murburn concept. Biomed Rev. 2018a; 29:89-97.

Manoj KM. Aerobic respiration: criticism of the proton-centric explanation involving rotary ATP synthesis, chemiosmosis principle, proton pumps and electron transport chain, Biochem Insights 2018b; 11:1178626418818442.

Manoj KM, et al. Murburn scheme for mitochondrial thermogenesis. Biomed Rev. 2018; 29:73-82.

Manoj KM, et al. Aerobic respiration: proof of concept for the murburn perspective. J Biomol Struct Dyn. 2019; 37:4524-4556.

Manoj KM, et al. Chemiosmotic and murburn explanations for aerobic respiration: predictive capabilities, structure-function correlations and chemico-physical logic. Arch Biochem Biophys. 2019; 676:108128.

Manoj KM, Bazhin N. Murburn precepts of aerobic respiration and homeostasis. OSF Preprints. (2019) doi:10.31219/osf.io/hx4p9

Manoj KM. Refutation of the cation-centric torsional ATP synthesis model and advocating murburn scheme for mitochondrial oxidative phosphorylation. Biophys Chem. 2020a; 257:106278.

Manoj KM. Murburn concept: a paradigm shift in cellular metabolism and physiology. Biomol Concepts 2020b; 11:7-22.

Manoj KM, et al. Acute toxicity of cyanide in aerobic respiration: Theoretical and experimental support for murburn explanation. Biomol. Concepts 2020a; 11:32-56.

Manoj KM, et al. What is the role of lipid membrane-embedded quinones in mitochondria and chloroplasts? Chemiosmotic Q-cycle versus burburn reaction perspective. Cell Biochem Biophys. (2020b) doi: 10.1007/s12013-020-00945-y

Manoj KM. Critical analysis of some assumptions and observations on photolytic oxygenesis by plant cells. OSF Preprints. 2020c. doi:10.31219/osf.io/y62j5

Manoj KM, et al. Is Z-scheme a tenable explanation for the light reaction of oxygenic photosynthesis? OSF Preprints. 2020c. doi:10.31219/osf.io/v6tdf

Manoj KM, et al. Microsomal xenobiotic metabolism, mitochondrial oxidative phosphorylation and cyanobacterial photorespiration have common murburn mechanistic underpinnings with chloroplastid photosynthetic physiology. OSF Preprints. 2020d. doi:10.31219/osf.io/8p2sx 
Manoj KM, et al. Murburn precepts for the light reaction of oxygenic photosynthesis. OSF Preprints. 2020e. doi:10.31219/osf.io/95brg

Mazor Y, Borovikova A, Caspy I, Nelson N Structure of the plant photosystem I supercomplex at $2.6 \AA$ resolution. Nat Plants 2017; 3:17014.

Messina F, Vella E, Cannas M, Boscaino R. Evidence of delocalized excitons in amorphous solids. Phys. Rev. Lett. 2010; 105:116401.

Nalbach J, Brawn D, Thorwart M. Exciton transfer dynamics and the quantumness of energy transfer in the Fenna-Matthew-Olson complex. Phys Rev E. 2011; 84:041926.

Otrin L, Marusic N, Bednarz C, et al. Toward artificial mitochondrion: Mimicking oxidative phosphorylation in polymer and hybrid membranes. Nano Lett. 2017; 17:6816-6821.

Panitchayangkoon G, Voronine D V, Abramavicius D, et al. Direct evidence of quantum transport in photosynthetic light-harvesting complexes. Proc Natl Acad Sci USA. 2011; 108:20908-20912.

Parashar A, Gade SK, Potnuru M, Madhavan M, Manoj KM. The curious case of benzbromarone: insight into super-inhibition of cytochrome P450. PLoS One 2014; 9:e89967.

Philipson KD, Sauer K. Exciton interaction in a bacteriochlorophyll-protein from Chloropseudomonas ethylica. Absorption and circular dichroism at 77 degrees K. Biochemistry 1972; 11:1880-1885.

Racker E, Stoeckenius W. Reconstitution of purple membrane vesicles catalyzing light-driven proton uptake and adenosine triphosphate formation. J Biol Chem. 1974; 249:662-663.

Romero E, et al. Quantum coherence in photosynthesis for efficient solar-energy conversion. Nature Phys. 2014; 10:676-682.

Rousso I, Friedman N, Sheves M, Ottolenghi M. $\mathrm{pK}_{\mathrm{a}}$ of the protonated Schiff base and aspartic 85 in the bacteriorhodopsin binding site is controlled by a specific geometry between the two residues.

Biochemistry 1995; 34:12059-12065.

Sarwar Jahan Md., et al. Light-harvesting complexes in photosystem II regulate glutathione-induced sensitivity of Arabidopsis guard cells to abscisic acid. J Plant Physiol. 2016; 195: 1-8.

Schindewolf U. Formation and properties of solvated electrons. Angew Chemie Int Edn 1988; 7:190-203.

Sheves M, Albeck A, Friedman N, Ottolenghi M. Controlling the $\mathrm{pK}_{\mathrm{a}}$ of the bacteriorhodopsin Schiff base by use of artificial retinal analogues. Proc Natl Acad Sci U S A. 1986; 83:3262-3266.

Steinberg G, Ottolenghi M, Sheves M. pKa of the protonated Schiff base of bovine rhodopsin: A study with artificial pigments. Biophys J. 1993; 64:1499-1502.

Takahashi NMI, Mikami N, Matsuda T, Miyamoto J. Identification of reactive oxygen species generated by irradiation of aqueous humic acid solution. J Pestic Sci. 1988; 13:429-435.

Tiwari V, Peters WK, Jonas DM. Electronic correlations with anticorrelated vibrations drive energy transfer outside the adiabatic framework. Proc Natl Acad Sci USA. 2013; 110:1203-1208.

Trchounian A, Petrosyan M, Sahakyan N. Plant cell redox homeostasis and reactive oxygen species. In: Gupta D, Palma J, Corpas F (eds) Redox state as a central regulator of plant-cell stress responses. Springer, Cham (2016)

Voet D, Voet JG. Biochemistry $4^{\text {th }}$ edition. 2011; Wiley, Hoboken, USA.

Wei X, Su X, Cao P, et al. Structure of spinach photosystem II-LHCII supercomplex at 3.2 A resolution. Nature. 2016;534(7605):69-74. 
$\mathrm{Xu}$ YH, Liu R, Yan L, et al. Light-harvesting chlorophyll a/b-binding proteins are required for stomatal response to abscisic acid in Arabidopsis. J Exp Bot. 2012; 63:1095-1106.

Young ID, Ibrahim M, Chatterjee R et al Structure of photosystem II and substrate binding at room temperature. Nature 2016; 540:453-457. 